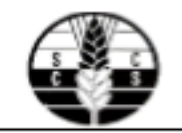

\title{
EFECTO DE LA MICORRIZACIÓN Y LA FERTILIZACIÓN FOSFORICA EN EL RENDIMIENTO DEL MAIZ (Zea mays L.) EN SUELOS ARENOSOS DE MONTERÍA
}

\section{Javier Arroyo $\mathbf{M}^{1}$ • $\bowtie$, German F. Estrella $\mathbf{C}^{2}$. y Eliécer Cabrales $\mathbf{H}^{3}$.,}

1 ingeniero Agrónomo,
CMSc. Grupo de
Investigación Agricultura
sostenible, Universidad de
Córdoba.
jamontiel26@gmail.com
2 ingeniero Agrónomo.
Grupo de Investigación
Agriculturarrastenible,
Universidad de Córdoba.
3 ingenieror Agrónomo.
PhD. Profesor área suelos,
Grupo de Investigación
Agriculturarrastenible,
Universidad de Córdoba.
PALABRAS r CLAVES:
Interpolación, fenología de la
planta, requerimiento hídrico,
manejo de riegos y manejo de
suelos.

\section{RESUMEN}

La prueba se llevó a cabo en el Corregimiento Buenos Aires, Montería Córdoba, cuyas coordenadas geográficas son $8^{\circ} 28^{\prime} 15^{\prime \prime} \mathrm{N}$ y $75^{\circ} 44^{\prime} 56^{\prime} \mathrm{O}$, en referencia al meridiano de Greenwich a 15 metros sobre el nivel del mar. El área pertenece a la zona climática cálida-moderada, la formación del Bosque Tropical Seco (BS-T) y la zona agroecológica Cj. Este estudio se llevó a cabo durante el segundo semestre del año 2018. El maíz de la variedad comercial (ICA V 109) se plantó a 0,5 x 0,8 m en un diseño de bloques completos al azar con un arreglo factorial $2 \times 5 \times 3$, tres repeticiones. Se evaluaron los parámetros de rendimiento. Se obtuvo que con el uso de micorrizas y dosis bajas de fósforo, se obtienen los mismos resultados que con dosis altas y sin micorrizas. La mejor dosis de $P$ aplicada al suelo en presencia de micorrizas es la de $50 \mathrm{~kg} / \mathrm{ha}$. Se concluye que, con el uso de micorrizas, la dosis de fósforo se puede reducir en el cultivo de maíz en condiciones edafoclimáticas de Buenos Aires - Montería y se mantienen los rendimientos, lo que contribuye a minimizar el impacto contaminante mediante el uso de fertilizantes de fósforo.

\section{EFFECT OF MICORRIZATION AND PHOSPHORIC FERTILIZATION ON THE PERFORMANCE OF CORN (Zea mays L.) IN SANDY SOILS OF MONTERÍA}

KEY WORDS:

mycorrhizae, phosphorus, yield mycorrhizal colonization, corn
SUELOS ECUATORIALES 49 (1 y 2$): 9-18$

ISSN 0562-5351 e-ISSN 2665-6558

\section{ABSTRACT}

The test was carried out in the Corregimiento Buenos Aires, Montería Córdoba whose geographical coordinates are $8^{\circ} 28^{\prime} 15^{\prime \prime} \mathrm{N}$ and $75^{\circ} 44^{\prime} 56^{\prime} \mathrm{O}$, referring to the Greenwich meridian at 15 meters above sea level. The area belongs to the warm-moderate climate zone, the Tropical Dry Forest (BS-T) formation and the $\mathrm{Cj}$ agroecological zone. This study was carried out during the second semester of the year 2018. Corn of the commercial variety (ICA V 109) was planted at $0.5 \times 0.8 \mathrm{~m}$ in a randomized complete block design with a $2 \times 5 \times 3$ factorial arrangement, three replications. Performance parameters were evaluated. It was obtained that with the use of mycorrhizae and low doses of phosphorus, the same results are achieved as with high doses and without mycorrhizae. The best dose of $P$ applied to the soil in the presence of mycorrhizae, is that of $50 \mathrm{~kg} / \mathrm{ha}$. It is concluded that, with the use of mycorrhizae, the dose of phosphorus can be reduced in the corn crop under edaphoclimatic conditions of Buenos Aires - Montería and the yields are maintained, which contributes to minimizing the contaminating impact by use of phosphorus fertilizers. 


\section{INTRODUCCIÓN}

Las micorrizas son organismos que han establecido una relación interespecífica con las plantas, lo que favorece el desarrollo de las mismas y la conservación y mejoramiento de características físicas del suelo, que a su vez incide en mejor aprovechamiento de los nutrientes del suelo, condiciones que conllevan a su uso por ser amigables con el medio ambiente. La importancia del uso de la simbiosis micorrízica radica en la absorción de agua y nutrientes para las plantas, esencialmente los de baja movilidad, como el fósforo, cobre y zinc. El primero cumple un papel importante en capacidad energética dentro de la planta y su ausencia puede incurrir en fisiopatías que desfavorecen el desarrollo de las plantas (Abdallah, 2014). Para el caso de las especies forrajeras, la deficiencia de fósforo en estos suplementos es condicionante para evitar desorden nutricional en el animal, ya que se afecta el crecimiento, brinda poco desempeño reproductivo, disminuye la producción de leche y la ganancia de peso (Cabrales et al, 2015), esto muestra la importancia de las micorrizas para la absorción de elementos de vital importancia para las plantas desde que el desarrollo metabólico induce crecimiento y desarrollo en estas, las micorrizas en la asociación simbiótica reciben de las plantas asimilados.

Las características físicas de los suelos de Buenos Aires, Montería son dadas a condiciones de texturas arenosas, lo que permite una fácil infiltración del agua y con esto una percolación de elementos nutricionales, lo que puede ser razón por lo que estos suelos son deficientes a nivel nutricional, ahora, estos mismos tienen altos contenidos de hierro lo que le propician las condiciones ácidas, sin prever en gran medida las condiciones de suelos de esta zona, la agricultura está arraigada a cultivos de pan coger, hortalizas y frutales, donde hay una especial acogida por el cultivo de maíz debido a caracteres culturales que han predominado en el tiempo, sin embargo, las labores de adecuación para este cultivo radican en labores culturales poco amigables con el ambiente tales como quemas, aplicación de herbicidas en forma desmesurada y no implementación de planes de fertilización para el desarrollo de cultivos, de tal modo que, la inmersión de estas prácticas no favorece 0 hace ineficiente el desarrollo de las simbiosis mutualistas del sueloplanta especialmente la micorrízica, precisando que la gran parte de estos cultivos son realizados por productores que poseen un conocimiento empírico en áreas que ocasionalmente superan una hectárea.

El fósforo en suelos tropicales tiene la particularidad de fijarse en distintas formas a la matriz coloidal del suelo, por lo cual, este elemento es considerado como uno de los principales problemas de importancia en la producción agrícola ya que gran parte de este no es soluble para las plantas, en base a esto, esta investigación tuvo como objetivo evaluar el efecto de las micorrizas y fertilización con distintas dosis de fósforo en el cultivo de maíz en condiciones edafoclimáticas de Buenos Aires, Montería.

\section{MATERIALES Y MÉTODOS}

Ubicación. El ensayo se llevó a cabo en el Corregimiento Buenos Aires, Montería Córdoba cuyas coordenadas geográficas son $8^{\circ} 28^{\prime} 15^{\prime \prime} \mathrm{N}$ y $75^{\circ} 44^{\prime} 56^{\prime \prime} \mathrm{O}$, en referencia al meridiano de Greenwich a $15 \mathrm{msnm}$. El área pertenece a la zona climática cálido-moderada, a la formación Bosque Seco Tropical (BS-T) y a la zona agroecológica Cj. Este estudio se realizó durante el segundo semestre del año 2018.

Muestreo de suelos. Se tomó una muestra de suelo compuesta en los primeros $20 \mathrm{~cm}$ de profundidad, de ella se empacó $1 \mathrm{~kg}$ aproximadamente y se rotuló, para realizar el respectivo análisis físico-químico.

Preparación del terreno y siembra. Se utilizó labranza cero, el lote se le hizo corte de vegetación. La siembra se hizo con maíz ICA V 109 , a $0.5 \times 0.8 \mathrm{~m}$. 
Inoculación micorrízica. Se aplicaron $5 \mathrm{~g}$ en cada hueco para siembra, sobre esta se colocó la semilla.

Manejo del cultivo. Para la nutrición de las plantas se implementó un plan de fertilización basado en lo siguiente: $150 \mathrm{~kg} / \mathrm{ha}$ de $\mathrm{N}$, esta dosis se distribuyó en 3 fracciones: $20-40-40 \%$ a los $15-$ 22-35 días después de emergido el cultivo, como fuente se utilizó la Urea. Para el K se aplicaron 90 $\mathrm{kg} / \mathrm{ha}$ de $\mathrm{K}_{2} \mathrm{O}$ y esta se aplicó en su totalidad al momento de la siembra, como fuente se utilizó el $\mathrm{KCl}$. La dosis de $\mathrm{P}_{2} \mathrm{O}_{5}$ correspondiente a cada tratamiento se aplicó también al momento de la siembra y en su totalidad, con la salvedad de aplicar solo la cantidad que corresponde según dosis en el tratamiento. Para los elementos menores, se trabajó con un fertilizante foliar comercial en dosis de $1 \mathrm{ml} /$ litro y se asperjó hasta humedecer toda la planta, se hizo dos aplicaciones: 15 y 30 días.

Evaluación micorrízica. Se hizo en base a la técnica de decoloración y tinción de raíces de Phillips y Hayman (1970) con algunas modificaciones.

Variables. Se evaluaron los parámetros fisiológicos (altura, área foliar, diámetro de tallo, masa seca, días a primera flor masculina y femenina) y los parámetros de rendimiento, de igual manera, se evaluó el grado de colonización.

Diseño estadístico. Se utilizó un diseño de bloques completos al azar con arreglo factorial, tres repeticiones $(2 \times 5 \times 3)$. La información se procesó con el programa estadístico SAS 9.0. se hizo ANAVA y comparación de medias por Tukey con una confiabilidad del $95 \%$.

\section{RESULTADOS Y DISCUSIONES}

Parámetros fisiológicos. Hubo diferencias significativas $p<0,05$ en el tratamientos micorrizado para los parámetros altura, diámetro de tallo, masa seca, donde las unidades experimentales con dosis de $50 \mathrm{~kg}$ de $\mathrm{P}_{2} \mathrm{O}_{5} / \mathrm{ha}$ ofertaron las mejores expresiones, estas mismas dosis obtuvieron floración masculina a los 43 días y la floración femenina a los 49,6 días después de siembra, mientras que la floración se presentó a los 50 días en las unidades experimentales con dosis de fósforo superiores a $50 \mathrm{~kg}$ de $\mathrm{P}_{2} \mathrm{O}_{5} / \mathrm{ha}$, mientras que el área foliar fue mayor en los tratamientos micorrizados con aplicación de $100 \mathrm{~kg}$ de $\mathrm{P}_{2} \mathrm{O}_{5} /$ ha Tabla 1 , lo que redunda en la formación de tejido vegetal cuando se aplica fósforo, siendo facilitada la absorción de este elemento por las micorrizas.

El tratamiento sin aplicación de micorrizas manifestó diferencias significativas $p<0,05$ para los componentes de crecimiento y desarrollo como altura, diámetro de tallo, en este tratamiento las dosis con mejor expresión fueron las unidades experimentales con $100 \mathrm{~kg}$ de $\mathrm{P}_{2} \mathrm{O}_{5} / \mathrm{ha}$, mientras que la primera floración masculina se dio a los 46,6 días después de siembra en las mencionadas dosis de $\mathrm{P}_{2} \mathrm{O}_{5}$, la primera flor femenina se presentó a los 49,6 días después de siembra en los tratamientos con dosis de 50 y $100 \mathrm{~kg}$ de $\mathrm{P}_{2} \mathrm{O}_{5} / \mathrm{ha}$ y la floración se presentó a los 53 días en el tratamiento con dosis de $100 \mathrm{~kg}$ de $\mathrm{P}_{2} \mathrm{O}_{5} /$ ha los resultados obtenidos contrastan con los reportes de Kaya y Putinella (2013), quienes reportan días a floración de a los 57 días, mismas expresiones que deben estar dadas por condiciones ambientales distintas y variedades diferentes de cultivar. 
Tabla 1. Parámetros fisiológicos del maíz micorrizado y fertilizado a distintas dosis de fósforo.

\begin{tabular}{|c|c|c|c|c|c|c|c|}
\hline $\begin{array}{c}\text { Dosis de } \\
\begin{array}{c}\mathrm{P}_{2} \mathrm{O}_{5} \\
(\mathrm{~kg} / \mathrm{ha})\end{array}\end{array}$ & Inoculación & $\begin{array}{c}\text { Altura } \\
(\mathrm{cm})\end{array}$ & $\begin{array}{c}\text { Diámetro } \\
\text { de tallo } \\
(\mathrm{cm})\end{array}$ & $\begin{array}{c}\text { Masa seca } \\
(\mathrm{g})\end{array}$ & $\begin{array}{c}\text { Área foliar } \\
\left(\mathrm{cm}^{2}\right)\end{array}$ & $\begin{array}{c}\text { Primera flor } \\
\text { masculina } \\
\left(\text { días}^{2}\right.\end{array}$ & $\begin{array}{c}\text { Primera flor } \\
\text { femenina } \\
(\text { días })\end{array}$ \\
\hline \multirow{2}{*}{0} & $\mathrm{M}+$ & $166.30^{\mathrm{B}}$ & $2.41^{\mathrm{B}}$ & $83.57^{\mathrm{BC}}$ & $2524.1^{\mathrm{BCD}}$ & $46.66^{\mathrm{AB}}$ & $46.33^{\mathrm{B}}$ \\
\cline { 2 - 8 } & $\mathrm{M}-$ & $81.70^{\mathrm{B}}$ & $1.54^{\mathrm{B}}$ & $36.55^{\mathrm{C}}$ & $474.4^{\mathrm{D}}$ & $47.0^{\mathrm{AB}}$ & $51.33^{\mathrm{A}}$ \\
\hline \multirow{2}{*}{25} & $\mathrm{M}+$ & $202.17^{\mathrm{A}}$ & $2.53^{\mathrm{A}}$ & $143.65^{\mathrm{AB}}$ & $2954.0^{\mathrm{BC}}$ & $46.0^{\mathrm{AB}}$ & $47.66^{\mathrm{AB}}$ \\
\cline { 2 - 8 } & $\mathrm{M}-$ & $175.67^{\mathrm{A}}$ & $2.55^{\mathrm{A}}$ & $81.42^{\mathrm{BC}}$ & $795.4^{\mathrm{CD}}$ & $48.0^{\mathrm{A}}$ & $51.33^{\mathrm{A}}$ \\
\hline \multirow{2}{*}{50} & $\mathrm{M}+$ & $222.50^{\mathrm{A}}$ & $2.80^{\mathrm{A}}$ & $208.75^{\mathrm{A}}$ & $3987.5^{\mathrm{B}}$ & $43.0^{\mathrm{B}}$ & $46.33^{\mathrm{B}}$ \\
\cline { 2 - 8 } & $\mathrm{M}-$ & $181.0^{\mathrm{A}}$ & $2.54^{\mathrm{A}}$ & $157.93^{\mathrm{AB}}$ & $687.5^{\mathrm{D}}$ & $46.66^{\mathrm{AB}}$ & $49.66^{\mathrm{AB}}$ \\
\hline \multirow{2}{*}{15} & $\mathrm{M}+$ & $217.33^{\mathrm{A}}$ & $2.64^{\mathrm{A}}$ & $204.11^{\mathrm{A}}$ & $4226.9^{\mathrm{B}}$ & $44.33^{\mathrm{AB}}$ & $46.33^{\mathrm{B}}$ \\
\cline { 2 - 8 } & $\mathrm{M}-$ & $182.0^{\mathrm{A}}$ & $2.51^{\mathrm{A}}$ & $101.33^{\mathrm{BC}}$ & $855.8^{\mathrm{CD}}$ & $46.66^{\mathrm{AB}}$ & $50.0^{\mathrm{AB}}$ \\
\cline { 2 - 8 } & $\mathrm{M}+$ & $206.50^{\mathrm{A}}$ & $2.78^{\mathrm{A}}$ & $150.80^{\mathrm{AB}}$ & $6482.4^{\mathrm{A}}$ & $45.33^{\mathrm{AB}}$ & $47.0^{\mathrm{B}}$ \\
\hline
\end{tabular}

M+: Inoculación micorrízica, M-: Sin inoculación micorrízica, letras diferentes en el superíndice en sentido vertical estadísticamente indican diferencias entre los tratamientos de acuerdo a la prueba de comparación de Tukey con un $95 \%$ de efectividad.

Así, se ha encontrado que las dimensiones de la altura en el cultivo de maíz incrementa a partir de los $40 \mathrm{~kg} / \mathrm{ha}$ (Mukhtar et al., 2018), resultados similares reporta Cruz (2017), quien acusa la no diferencia en alturas para el cultivar de maíz cuando evaluó distintas dosis de fertilizantes entre estos el fósforo, en este mismo sentido Drissi et al., (2015) para la variable diámetro de tallo reporta promedios encontrados para este mismo cultivar que no superan los 2,54 cm indicando diferencias significativas $\mathrm{p}<0,05$ al fertilizar con distintas dosis de $\mathrm{P}_{2} \mathrm{O}_{5}$, Olusegun, (2015) manifiesta diferencias estadísticas en diámetro de tallo, donde entraron valores medios máximos de $2,92 \mathrm{~cm}$ cuando fertilizó al cultivo de maíz con dosis crecientes de $\mathrm{P}_{2} \mathrm{O}_{5}$, siendo la mejor dosis cuando se aplicó $45 \mathrm{~kg} / \mathrm{ha}$.

Cuando las plantas sufren estrés por fósforo, se ha manifestado en el cultivo de maíz disminución su diámetro de tallo generando consigo mismo la disminución de células epidérmicas e hipodérmicas haciéndose más delgado, esto ocasiona haces vasculares más pequeños y de igual forma disminuye la cantidad de estos (Sarker et al., 2010), de acuerdo a la cantidad de fósforo aprovechable en el suelo, a medida que se incrementa la disponibilidad en la solución del suelo de este elemento también incrementa la absorción por la planta y por ende la biomasa seca (Pedersen et al., 2018), en esta misma variable se puede alcanzar hasta $200 \mathrm{~g}$ a los 60 días después de siembra (Gaviria, 2016), en el cultivo de maíz ICA V 109 se obtuvo valores simultaneos, bajo esta mismas condiciones, los resultados obtenidos son similares a los reportados por Amanullah et al (2016) en suelos con textura arenosa.

Componente de rendimiento. Hubo diferencias estadísticas $\mathrm{p}<0,05$ para los tratamientos evaluados, los rendimientos de las plantas micorrizadas fue mayor en comparación con las plantas donde no se inoculó micorrizas como se muestra en la gráfica 1, siendo la dosis de fósforo de $50 \mathrm{~kg}$ de $\mathrm{P}_{2} \mathrm{O}_{5} /$ ha la que mayor influenció en el rendimiento de las plantas de maíz, de las cuales se estimó un rendimiento de $8914 \mathrm{~kg}$, seguido por el tratamiento con dosis de fósforo aplicadas a razón de $75 \mathrm{~kg}$ de $\mathrm{P}_{2} \mathrm{O}_{5} / \mathrm{ha}$ donde se estimó un rendimiento de 8731.3 $\mathrm{kg} / \mathrm{ha}$, mientras que las plantas donde se 
estimó menor rendimiento fue donde no se aplicó fósforo. En contraste con las plantas donde no se inoculó micorrizas, se estimó un máximo rendimiento de $3656.6 \mathrm{~kg} / \mathrm{ha}$ cuando se aplicó $50 \mathrm{~kg}$ de $\mathrm{P}_{2} \mathrm{O}_{5} / \mathrm{ha}$, mientras que el menor rendimiento se obtuvo cuando no se aplicó fósforo donde se estimó $300 \mathrm{~kg} / \mathrm{ha}$ para las plantas evaluadas.

Gráfica 1. Rendimiento del maíz micorrizado y fertilizado con distintas dosis de fósforo.

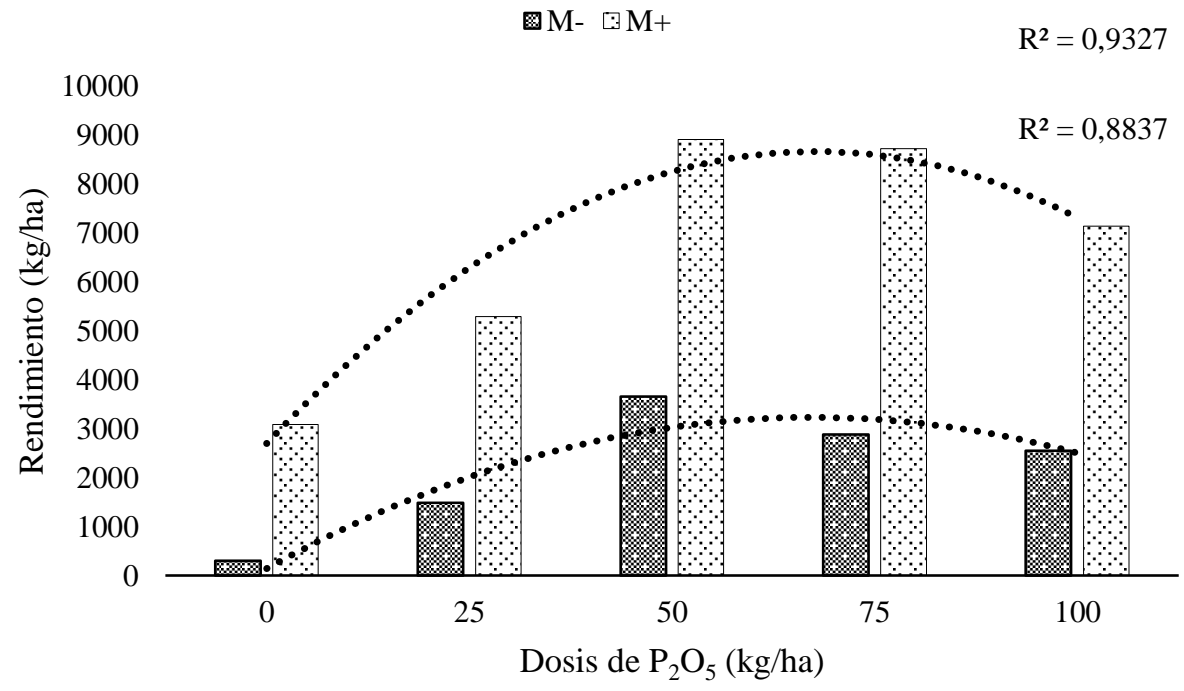

M+: Inoculación micorrízica, M-: Sin inoculación micorrízica.

Tabla 2. Parámetros de rendimiento del maíz micorrizado y fertilizado a distintas dosis de fósforo

\begin{tabular}{|c|c|c|c|c|c|c|}
\hline $\begin{array}{c}\text { Dosis de } \\
\mathrm{P}_{2} \mathrm{O}_{5} \\
(\mathrm{~kg} / \mathrm{ha})\end{array}$ & Inoculación & $\begin{array}{c}\text { Número de } \\
\text { mazorcas/sitio }\end{array}$ & $\begin{array}{l}\text { Longitud de } \\
\text { mazorcas }(\mathrm{cm})\end{array}$ & $\begin{array}{c}\text { Número de } \\
\text { hileras/mazorca }\end{array}$ & $\begin{array}{l}\text { Granos por } \\
\text { hilera }\end{array}$ & $\begin{array}{c}\text { Peso de } \\
100 \text { granos } \\
(\mathrm{g})\end{array}$ \\
\hline \multirow{2}{*}{ 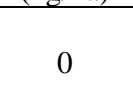 } & $\mathrm{M}+$ & $3.0^{\mathrm{ABC}}$ & $15.08^{\mathrm{A}}$ & $12.02^{\mathrm{A}}$ & $28.92^{\mathrm{A}}$ & $20.34^{\mathrm{A}}$ \\
\hline & M- & $0.5^{\mathrm{C}}$ & $2.66^{\mathrm{B}}$ & $4.0^{\mathrm{B}}$ & $6.0^{\mathrm{B}}$ & $6.16^{\mathrm{B}}$ \\
\hline \multirow{2}{*}{25} & M+ & $3.22^{\mathrm{AB}}$ & $12.38^{\mathrm{A}}$ & $12.88^{\mathrm{A}}$ & $25.66^{\mathrm{A}}$ & $17.98^{\mathrm{A}}$ \\
\hline & M- & $1.33^{\mathrm{BC}}$ & $12.0^{\mathrm{A}}$ & $12.83^{\mathrm{A}}$ & $21.0^{\mathrm{AB}}$ & $17.44^{\mathrm{A}}$ \\
\hline \multirow{2}{*}{50} & M+ & $4.0^{\mathrm{A}}$ & $15.14^{\mathrm{A}}$ & $13.97^{\mathrm{A}}$ & $32.02^{\mathrm{A}}$ & $21.96^{\mathrm{A}}$ \\
\hline & M- & $2.77^{\mathrm{ABC}}$ & $12.96^{\mathrm{A}}$ & $13.04^{\mathrm{A}}$ & $24.35^{\mathrm{A}}$ & $16.64^{\mathrm{AB}}$ \\
\hline \multirow{2}{*}{75} & M+ & $4.11^{\mathrm{A}}$ & $15.62^{\mathrm{A}}$ & $13.60^{\mathrm{A}}$ & $34.33^{\mathrm{A}}$ & $21.66^{\mathrm{A}}$ \\
\hline & M- & $2.88^{\mathrm{ABC}}$ & $12.3^{\mathrm{A}}$ & $13.11^{\mathrm{A}}$ & $25.51^{\mathrm{A}}$ & $19.19^{\mathrm{A}}$ \\
\hline \multirow{2}{*}{100} & M+ & $4.11^{\mathrm{A}}$ & $15.5^{\mathrm{A}}$ & $12.86^{\mathrm{A}}$ & $33.53^{\mathrm{A}}$ & $22.36^{\mathrm{A}}$ \\
\hline & M- & $2.44^{\mathrm{ABC}}$ & $13.28^{\mathrm{A}}$ & $13.36^{\mathrm{A}}$ & $22.66^{\mathrm{A}}$ & $18.92^{\mathrm{A}}$ \\
\hline
\end{tabular}

M+: Inoculación micorrízica, M-: Sin inoculación micorrízica, letras diferentes en el superíndice en sentido vertical estadísticamente indican diferencias entre los tratamientos de acuerdo a la prueba de comparación de Tukey con un $95 \%$ de efectividad.

También para las variables derivadas del rendimiento Tabla 2 presentaron diferencias estadísticas p<0.05 así, el número de mazorcas en el tratamiento micorrizado fue 
mayor cuando se incrementó las dosis de $\mathrm{P}_{2} \mathrm{O}_{5}$ cuando se aplicó dosis de este elemento de 75 $\mathrm{kg} / \mathrm{ha}$ y $100 \mathrm{~kg} / \mathrm{ha}$ mientras que cuando no se inoculó micorrizas el mayor número de mazorca se obtuvo cuando se aplicó $75 \mathrm{~kg}$ de $\mathrm{P}_{2} \mathrm{O}_{5} /$ ha, en este sentido, la longitud de mazorcas en las plantas donde hubo inoculación de micorrizas fue mayor cuando se aplicó $75 \mathrm{~kg}$ de $\mathrm{P}_{2} \mathrm{O}_{5} / \mathrm{ha}$, efectuando un crecimiento ascendente a medida que incrementa las dosis de fósforo, pero en las plantas sin inoculación de micorrizas la longitud de las mazorcas en promedio fue mayor cuando se aplicó $100 \mathrm{~kg}$ de $\mathrm{P}_{2} \mathrm{O}_{5} /$ ha, mientras que el número de hileras fue mayor con la aplicación de $50 \mathrm{~kg}$ de $\mathrm{P}_{2} \mathrm{O}_{5} /$ ha, aunque la cantidad de granos por hilera presentó su máxima expresión en las plantas micorrizadas y fertilizadas con $75 \mathrm{~kg}$ de $\mathrm{P}_{2} \mathrm{O}_{5} / \mathrm{ha}$, dando las dosis de fósforo de $100 \mathrm{~kg}$ de $\mathrm{P}_{2} \mathrm{O}_{5} / \mathrm{ha}$ la mayor expresión de peso de 100 granos.

Los resultados obtenidos superan los reportados por Cabrales et al.,(2016) cuando evaluó distintos tipos de micorrizas en el cultivo de maíz en el estado Guárico (Venezuela) bajo condiciones de suelos ácidos, así, se ha reportado que las no aplicaciones de fósforo se obtiene en promedio 0,86 mazorcas (Masood, 2011), en el cultivo de maíz se halló similar tendencia en su colonización micorrízica cuando se evaluó disitntas dosis de fósforo, en tal forma Kumari et al., (2018) cuando utilizó como fuente de fósforo $\mathrm{P}_{2} \mathrm{O}_{5}$ en dosis crecientes desde el tratamiento testigo sin aplicación hasta $80 \mathrm{~kg} / \mathrm{ha}$ encontró que en esta última dosis se obtuvo 1,41 mazorcas por planta, pero Ravi et al., (2018), evaluó dosis de $\mathrm{P}_{2} \mathrm{O}_{5}$ hasta $75 \mathrm{~kg} / \mathrm{ha}$ y obtuvo 1,03 mazorcas por planta en la máxima dosis de fósforo aplicado, para la longitud de mazorcas Subaedah et al., (2016) quien al evaluar dosis de $\mathrm{P}_{2} \mathrm{O}_{5}$ hasta 66 $\mathrm{kg} / \mathrm{ha}$ incluido el testigo sin aplicación de este elemento obtuvo mazorcas con longitudes promedio entre $18,30 \mathrm{~cm}-20,27 \mathrm{~cm}$, en dosis de fósforo a razón de $100 \mathrm{~kg} / \mathrm{ha}$ usado como compuesto el $\mathrm{P}_{2} \mathrm{O}_{5}$ Khan (2012) se obtuvo longitudes de mazorca mínima de 20,06 cm y la máxima expresión fue dada en 21,12 cm.

En el cultivo de maíz bajo fertilización de fósforo Banotra et al., (2017) quien evaluó dosis de $\mathrm{P}_{2} \mathrm{O}_{5}$ a razón $60 \mathrm{~kg} / \mathrm{ha}$ obtuvo 13,29 hileras por mazorca, Singh et al., (2018) encontró ante la aplicación de 28,98 kg de fósforo la expresión de 13,72 hileras por mazorca, Asim et al., (2017) al evaluar la proporción de $90 \mathrm{~kg}$ de $\mathrm{P}_{2} \mathrm{O}_{5} /$ ha usando como fuente DAP, alcanzó en el cultivo de maíz 33,81 granos por hilera de mazorca, similar al valor obtenido cuando se aplicó $100 \mathrm{~kg}$ de $\mathrm{P}_{2} \mathrm{O}_{5} /$ ha, pero no se supera los 38 granos aproximados que reporta Martínez et al (2018) en el cultivo de maíz desarrollado bajo condiciones de fertilización fosfórica, , Kalhapure (2013) informa que en dosis de 60 $\mathrm{kg}$ de $\mathrm{P}_{2} \mathrm{O}_{5} / \mathrm{ha}$ los 100 granos pesaron $24,9 \mathrm{~g}$ en promedio, de igual forma reporta Martínez et al., (2018) pesos mínimos de 25,2 g y máximos de 26,2 g ante la aplicación de fertilización sintética que incluía al fósforo y Pal et al., (2017) halló en el peso de 100 granos en el desarrollo del cultivo de maíz 25,27 g en el peso de 100 granos cuando aplicó $\mathrm{P}_{2} \mathrm{O}_{5}$ a razón de $60 \mathrm{~kg} / \mathrm{ha}$,

Colonización. Para la colonización micorrízica no hubo diferencias estadísticas significativas $\mathrm{p}>0,05$ pero la gráfica 2 , muestra que la colonización fue ligeramente mayor en el tratamiento con inoculo de micorrizas y dosis de $25 \mathrm{~kg}$ de $\mathrm{P}_{2} \mathrm{O}_{5} /$ ha donde se dio una colonización del 95,01\% mientras que la colonización menor se dio donde no se inoculó micorrizas y se aplicó dosis de fósforo a razón de $100 \mathrm{~kg}$ de $\mathrm{P}_{2} \mathrm{O}_{5} / \mathrm{ha}$, en esta comparación de tratamiento se muestra que la colonización nativa del suelo estudiado en Buenos Aires-Montería varía ligeramente en un $10 \%$ por debajo de los tratamientos inoculados con micorrizas. 
Gráfica 2. Colonización micorrízica del maíz micorrizado y fertilizado con distintas dosis de fósforo.

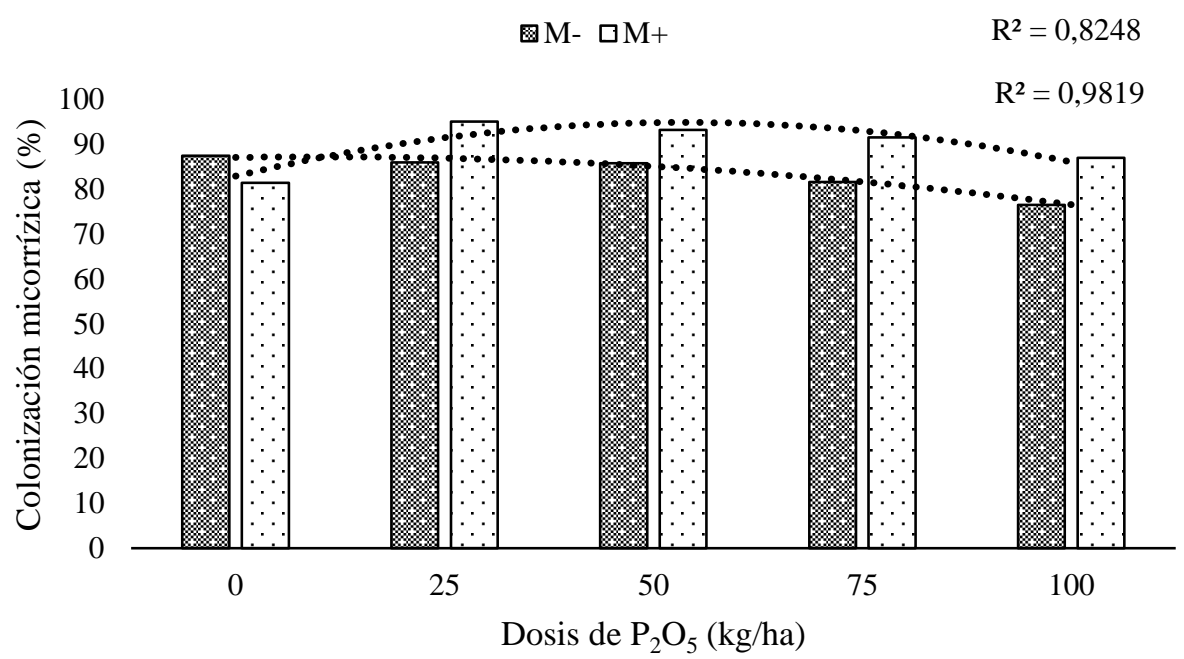

M+: Inoculación micorrízica, M-: Sin inoculación micorrízica.

El componente de colonización micorrízica para los tratamientos evaluados acusa la presencia de micorrizas nativas en los suelos del corregimiento Buenos Aires toda vez que el tratamiento sin inoculación de micorrizas presentó una colonización de $87,36 \%$ en las unidades experimentales sin aplicación de fósforo, esto posiblemente obedece a los mecanismos de perpetuación de especies donde las plantas al sufrir estrés por falta de nutrientes se efectúa la relación interespecífica mutualista entre planta y micorrizas. Donde se hizo inoculación con micorrizas se lograron colonizaciones de hasta del 95\% cuando se aplicaron $25 \mathrm{~kg}$ de $\mathrm{P}_{2} \mathrm{O}_{5} /$ ha, esto resultados concuerda con los reportes de Cabrales (2015G), quien encontró que dosis bajas de $\mathrm{P}$ indicen a la colonización micorrízica, iguales resultados reportan (Seguel et al; 2012) en suelos ácidos, Sawers et al., (2016) quien obtuvo porcentajes de infección micorrízica variables entre 69,2\% $96,8 \%$ en el cultivo de maíz, para la evaluación hecha en las plantas sin inoculación micorrízica se observó que la micorrización disminuyó a medida que incrementó las dosis de fósforo, estos mismos resultados concuerdan con la tendencia de colonización que halló Wang et al, (2017) cuando evaluó el fósforo hasta los $100 \mathrm{~kg} / \mathrm{ha}$ de aplicaciones de fósforo sintético en la formación de estructuras de infección micorrízica, por lo cual, debe suponerse que la afectación micorrízica posiblemente sea problema cuando se aplica fósforo ante colonizaciones nativas o que se encuentren adaptadas ecológicamente en un ambiente definido, así mismo, Sarabia et al., (2017) informa que mientras no se hace aplicación de fósforo sintético la infección micorrizal se incrementa hasta el $64 \%$ en plantas de maíz, de tal forma que Farias et al., (2018) ha encontrado resultados del $69,4 \%$ en este mismo cultivo si realizar una inoculación previa. 


\section{CONCLUSIONES}

La inoculación micorrízica es una alternativa para incrementar la producción del cultivo de maíz en suelos arenosos y de baja oferta nutricional en Buenos Aires- Montería, ofertando el mejor carácter fisiológico y de rendimiento cuando se aplican dosis de $50 \mathrm{~kg}$ de $\mathrm{P}_{2} \mathrm{O}_{5} /$ ha.

La colonización micorrízica se incrementa con bajas dosis de fósforo en el cultivo de maíz cuando, siendo la mejor dosis para lograr una buena colonización hasta $25 \mathrm{~kg}$ de $\mathrm{P}_{2} \mathrm{O}_{5} /$ ha.

\section{REFERENCIAS}

Abdallah, A. 2014. Effect of diammonium phosphate fertilization on growth and yield of irrigated forage maize (Zea mays L.). Thesis submitted in partial fulfillment for the Degree of M.Sc (Agronomy). Sudanuniversity of Science and Technolog College of Graduate Studies, Sudan.

Aguilera, L., Olalde, V., Arriaga, M. y Contreras, R. 2007. Micorrizas arbusculares. Ciencia Ergo Sum 14(3):300-306.

Amanullah, Saleem, A., Iqbal, A y Fahad, S. 2016. Foliar Phosphorus and Zinc application improve growth and productivity of maize (Zea mays L.) under moisture stress conditions in semi-arid climates. Journal microbial y biochemical technology 8(5):433439.

Asim, M., Hussain, Q., Ali, A., Farooq, S., Khan, R. and Ali, S. 2017. Responses of maize to differnt levels and sources of phosphorus application. Pure and Applied Biology 6(3):1030-1036.

Banotra, M., Sharma B., Nandan B., Verma A., Shah, I., Kumar, R., Gupta, V and Namgial. 2017. Growth, Phenology, Yield and Nutrient uptake of sweet corn as influenced by cultivars and planting times under irrigatd subtropics of Shiwalik foot hills. International Journal of Current Microbiology and Applied Sciences 6(10):2971-2985.

Cabrales, E., Toro, M y Lopez D. 2016. Efecto de micorrizas nativas y fósforo en los rendimientos del maíz en Guárico, Venezuela. Temas Agrarios 21(2):21-31.

Cabrales, E., Estrella, G y Vásquez, E. 2015 Evaluación comparativa de poblaciones de nematodos de tres pasturas en el Bajo CaucaColombia. Suelos Ecuatoriales 45(2):65-71.

Drissi, S., Aït, A., Bamouh, A., Coquant, J y Benbella, M. 2015. Effect of Zinc-Phosphorus interaction on corn silage grown on Sandy soil. Agriculture 5(4):1047-1059.

eFarias, C., de Carvalho, R., Resende, F. and Azevedo, L. 2018. Consortium of five fungal isolates conditioning root growth and arbuscular mycorrhiza in soybean, corn, and sugarcane. Anais da Academia Brasileira de Ciências 90(4): 3649-3660.

Gaviria, B. 2016. Análisis de la fenología e índices de crecimiento de maíz (Zea mays L.) variedad pioneer, Curn-Armero Tolima. En línea:

https://www.researchgate.net/publication/322 477901_ANALISIS_DE_LA_FENOLOGIA EE_INDICES_DE_CRECIMIENTO_DE_M AIZ_Zea_Mays_1_VARIEDAD_PIONEER_ CURDN-

ARMERO_TOLIMA?enrichId=rgreq9b3a4e8435bccbf8b0c74cf614536bcbXXX\&enrichSource $=Y 292 Z X J Q Y W d l O z M$ yMjQ3NzkwMTtBUzo1ODI0NTMxNzYw MTI4MDBAMTUxNTg3OTA2NjA3OQ\%3 D\%3D\&el=1_x_2\&_esc=publicationCoverP df [27 nov. 18]. 
Kalhapure, A., Shete, B. and Dhonde, M. 2013. Integrated nutrient management in maize (Zea mays L.) for increasing production with sustainability. International Journal of Agriculture and Food Science Technology 4(3):195-206.

Kaya, E y Putinella, J. 2013.effect of sago palm waste compost and SP-36 fertilizer against the availability of $\mathrm{P}, \mathrm{P}$ uptake and growth of corn (Zea mays L.) in Ultisols. International Journal of Agronomy and Agricultural Research (IJAAR) 3(11):16-22.

Khan, W., Singh, V., Sagar, A., and Sachchida, N. 2017. Response of phosphorus application on growth and yield attributes of sweet corn (Zea mays L. saccharata) varieties. Journal of Pharmacognosy and Phytochemestry 6(5):2144-2146.

Kumari, E., Sen, A., Maurya, B., Sarma., B., and Upadhyay, P. 2018. Effect of different microbial strains on growth parameters viz. Lai, Cgr, Rgr and Nar of baby corn. Journal of Pharmacognosy and Phytochemistry

7(2):3037-3040.

Martínez, L., Aguilar, C., Carcaño, M., Galdámez, J., Gutiérrez, A., Morales, J., Martínez, F., Llaven, J., y Gómez E. 2018. Biofertilización y fertilización química en maíz (Zea mays L.) en Villaflores, Chiapas, México. Siembra 5(1):26-37.

Masood, T., Gul, R., Munsif, F., Jalal, F., Hussain, Z., Noreen, N., Khan, H., Nasiruddin and Khan, H. efecto of different phosphorus levels on the yield and yield components of maize. Sarhad Journal of Agriculture 27(2):167-170.

Mukhtar, T., Arif, M., Hussain, S., Tariq, M. and Mehmood, K. 2011. Effect of different rates of nitrogen and phosphorus fertilizers on growth and yield of maize. Journal of Agricultural Research 49(3):33-339.
Olusegun, O. 2015. Nitrogen (N) and Phosphorus $(\mathrm{P})$ fertilizer application on maize (Zea mays L.) growth and yield at Ado-Ekiti, South-West, Nigeria. American Journal of Experimental Agriculture 6(1):22-29.

Pal, B., Hirpara, D., Vora, V., Vekaria, P., Sutaria, G., Akbari, K. and Verma, H. 2017. Effect of nitrogen and phosphorus on yield and yiel attributes of maize in South Saurashtra, India. International Journal of Current Microbiology and Applied Science 6(3):1945-1949.

Palencia, G., Mercado, T. y Combatt, E. 2006. Estudio Agroclimático del Departamento de Córdoba. Montería: Universidad de Córdoba. 126p.

Phillips, J. M., and S. D. Hayman. 1970. Improved procedures for clearing roots and staining parasitic and vesicular-arbuscular mycorrhizal fungi for rapid assessment of infection. Trans. Brit. Mycol. Soc. 55: 158161.

Pedersen, I., Sorensen, P., Christensen, B y Rubæk, G. 2018. Do phosphorus and nitrogen contents in young corn leaves represent contents in shoots?. Canadian Journal of Plant Science 98(5):1199-1202.

Ravi, U., Munirathnam, P., Prabhakara, G., y Kavitha, P. 2018. Standardization of sowing window and phosphorus requirement for Kharif maize (Zea mays L) in vertisols. 2018. International Journal of Pure and Applied Bioscience 6(5):754-759.

Sarabia, M., Cornejo, P., Azcón, R., Carreón, Y. and Larsen, J. 2017. Mineral phosphorus fertilization modulates interactions between maize, rhizosphere yeasts and arbuscular mycorrhizal fungi. Rhizosphere 4:89-93.

Sarker, B., Karmoker, J y Rashid, P. effects of phosphorus deficiency on anatomical 
structures in maize (Zea mays L.). Bangladesh Journal of Botany 39(1):57-60.

Sawers, R., Svane, S., Quan, C., Grønlund, M., Wozniak, B., Nigussie, M., González, E., Chávez, R., Baxter, I., Goudet, J., Jakobsen, I. and Paszkowski, U. 2016. Phosphorus acquisition efficiency in arbuscular mycorrhizal maize is correlated with the abundance of root-external hyphae and the accumulation of transcripts encoding PHT1 phosphate transporters. New Phytologist 214:632-643.

Seguel, A., Medina, J., Rubio, R., Cornejo, P., and Borie, F. 2012. Efects of soil aluminum on early arbuscular mycorrhizal colonization of wheat and barley cultivars growing in an andisol. Chilean Journal of Agricultural Research; 72(3) 449-455.

Singh, S., Singh, V., Datt, R., y Singh, K. 2018. Effect of fertilizer levels and biofertilizer on Green cob yield of corn (Zea mays L.). International Journal of Chemical Studies 6(2):2188-2190.

Subaedah, S., Aladin, A y Nirwana. Fertilization of nitrogen, phosphor and application of green manre of Crotalaria juncea in increasing yield of maize in marginal dry land. Agriculture and Agricultural Science Procedia 9(2016):20-25.

Wang, C, White, P and Li, C. 2017. Colonization and community structure of arbuscular mycorrhizal fungi in maize roots at different depths in the soil profile respond differently to phosphorus inputs on a longterm experimental site. Mycorrhizal 27(4):369-381. 